\title{
(1) \\ Distribución de vacuna COVID-19: Combinando SEIR y Machine Learning
}

\author{
COVID-19 Vaccine Distribution: Combining SEIR and Machine Learning
}

Distribuição de vacinas COVID-19: Combinando SEIR e Machine Learning

Víctor López-Sandoval ${ }^{1}$

Received: Mar/17/2021 • Accepted: Jul/15/2021 • Published: Jan/31/2022

\section{Resumen (10)}

Este estudio tiene como objetivo general construir un modelo epidémico con control por vacunación para el Covid-19 en El Salvador. Se propone la combinación de modelos epidemiológicos SEIR (Susceptibles, Expuestos, Infectados o Recuperados) y la estimación de parámetros usando machine learning y redes de contacto. El proyecto se desarrolló siguiendo tres fases: a) Análisis: se realizó la identificación de factores o variables críticas o claves del fenómeno en estudio, se definió, diseñó y construyó el modelo a utilizar junto con sus parámetros y componentes. b) Simulación: una vez construido el modelo, se desarrolla una simulación de este. La simulación permitió modificar variables, implementar alternativas y hacer modificaciones al modelo sin afectar al sistema real, lo cual es de gran utilidad en la toma de decisiones y elaboración de resultados y recomendaciones. Se desarrollan las simulaciones con datos poblacionales de El Salvador. c) Optimización: se evaluaron diferentes escenarios en los cuales se aplican medidas de control por vacunación y medidas de distanciamiento social, con el objetivo de identificar la estrategia óptima. Como resultado del estudio se identificó como mejor estrategia para el control de la enfermedad: vacunar a la población vulnerable y mantener medidas de distanciamiento social, la combinación de estas dos políticas brindó los mejores resultados en función de disminuir el impacto de la infección y de minimizar los costos del tratamiento. Al final, se brindan recomendaciones a las autoridades de salud gubernamentales para la distribución y aplicación del tratamiento.

Palabras clave: SEIR; Machine Learning; Modelo Epidemiológico; Vacunación; COVID-19; El Salvador

\section{Abstract (iD)}

The purpose of this study is to build an epidemic model with vaccination control for Covid-19 in El Salvador. A combination of epidemiological SEIR (Susceptible, Exposed, Infectious or Recovered) models and the estimation of parameters using machine learning and contact networks is proposed. The project consisted of three phases: a) Analysis: the critical or key factors or variables of the phenomenon under study were identified, the model to be used, as well as its parameters and components, were defined, designed, and constructed b) Simulation: simulation made it possible to modify variables, implement alternatives, and modify the model itself without affecting the real system, which is highly useful for decision-making and preparing results and recommendations. The simulations were carried out using population data from El

Víctor López-Sandoval, \ victorlopez@ugb.edu.sv, (D) https://orcid.org/0000-0001-8661-8976

1 Vicerrectoría de Investigación, Universidad Gerardo Barrios, San Miguel, El Salvador 
Salvador. c) Optimization: different scenarios were evaluated in which vaccination control measures and social distancing measures were applied, in order to identify the optimal strategy. As a result of this study, the best strategy for controlling the disease was identified: a combination of vaccinating the vulnerable population and maintaining social distancing measures provided the best results in terms of reducing the impact of infection and minimizing treatment costs. Finally, recommendations are made to government health authorities for distribution and application of the treatment.

Keywords: SEIR; Machine Learning; Epidemic Model; Vaccination; COVID-19; El Salvador

\section{Resumo}

Este estudo tem como objetivo geral construir um modelo epidêmico com controle por vacinação para a Covid-19 em El Salvador. Propõe-se a combinação de modelos epidemiológicos SEIR(Suscetíveis, Expostos, Infectados ou Recuperados) e a estimativa de parâmetros utilizando machine learning e redes de contato. 0 projeto foi desenvolvido a partir de três fases: a) Análise: foi realizada a identificação de fatores ou variáveis críticas ou chave do fenômeno em estudo, o modelo a ser utilizado foi definido, desenhado e construído juntamente com seus parâmetros e componentes. b) Simulação: uma vez que o modelo é construído, uma simulação dele é desenvolvida. A simulação permitiu modificar variáveis, implementar alternativas e fazer modificações no modelo sem afetar o sistema real, o que é muito útil na tomada de decisão e na elaboração de resultados e recomendações. Simulações são desenvolvidas com dados populacionais de El Salvador. c) Otimização: foram avaliados diferentes cenários em que são aplicadas medidas de controle de vacinação e medidas de distanciamento social, com o objetivo de identificar a estratégia ideal. Como resultado do estudo foi identificada como a melhor estratégia para o controle da doença: vacinar a população vulnerável e manter medidas de distanciamento social, a combinação dessas duas políticas proporcionou os melhores resultados em termos de redução de impacto da infecção e minimização dos custos do tratamento. No final, são fornecidas recomendações às autoridades governamentais de saúde para a distribuição e a aplicação do tratamento.

Palavras-chave: SEIR; machine learning; modelo epidemiológico; vacinação; COVID-19; El Salvador

\section{Introducción}

La llegada de una vacuna frente al SARS-CoV-2 que pueda frenar la expansión de la infección se ha postulado como la respuesta definitiva para frenar la pandemia de COVID19 (Piqueras, Carmona, y Bernáldez, 2020). Se espera que para el año 2021, existan alternativas para inmunizar la población ante esta enfermedad y poder paulatinamente, regresar a la normalidad. Cuando esa vacuna llegue, los gobiernos tendrán que implementar los programas de vacunación que permitan alcanzar los niveles de inmunización necesarios para evitar la transmisión de la enfermedad (González-Melado y Di Pietro, 2020). Por el momento (enero de 2020), once vacunas están en la fase 3, previa a la comercialización (WHO, 2021).

En el devenir histórico, dentro del estudio formal de las epidemias, se han desarrollado diferentes estudios con el objeto de analizar enfermedades infecciosas relacionadas con la influenza y sus medidas de control, estos estudios se documentan 
en (Chowell et al. 2006), (Acuña, Castañeda y Chowell, 2011), (Arino, Brauer y den Driessche, 2018), (Herrera, Cruz, y Castillo-Chavez, 2011), (Nuño, Chowell y Castillo-Chavez, 2007). Algunos de estos estudios incluyen por ejemplo el impacto del transporte, el uso de medidas de control como distanciamiento social o la dinámica de la enfermedad través de sistemas de ecuaciones diferenciales. Estos estudios han abonado en gran medida a la comprensión de los fenómenos pandémicos y sirven de base para el análisis formal. En este proyecto, se pretende combinar estos modelos teóricos que han nacido desde una perspectiva matemática, con herramientas de machine learning y tecnología actual, lo cual a priori, genera una nueva herramienta para el análisis de la dinámica de vacunación de la población.

Se ha vuelto necesario establecer una propuesta técnica que sirva para guiar el proceso, pues, sin ella estaremos condenados a las consecuencias de las respuestas tardías y la dependencia de las buenas intenciones de otras naciones y empresas privadas a compartir sus desarrollos tecnológicos (Gómez, 2020).

El control óptimo de forma clásica se puede resolver usando una versión discreta del principio del máximo de Pontryagin (Brauer et al. 2015) y (Lenhart y Workman, 2007), sin embargo, es importante construir un modelo basado en tecnologías emergentes que nos permita establecer criterios de distribución de manera fluida. Este tipo de propuestas tienen una gran utilidad para la población, ya que se implementa la toma de decisiones en temas de salud pública sobre criterios científicos y no por simples juicios de valor.

La investigación permitió combinar sistemas epidemiológicos clásicos usando ecuaciones diferenciales como los usados en (Saralegui, 2016), con métodos tecnológicos de punta, usando algoritmos de machine learning como los usados por (Chimmula y Zhang, 2020) y (Dal Molin, Gomes Da Silva, Cocco Mariani y Dos Santos, 2020)

\section{Metodología}

\section{Fase de Análisis}

En esta fase se realizó la identificación de los factores y variables del modelo. También se hizo la recopilación de datos para las diversas mediciones formales. En este apartado se da respuesta a dos grandes interrogantes:

1. ¿Cuál vacuna se ha seleccionado para el desarrollo de este estudio?

2. ¿Cuál es el modelo que se implementa para el desarrollo del proyecto?

Para contestar la primera pregunta, se considera que según la (OPS, 2020) para poder administrar una vacuna confiable, existen diferentes fases de desarrollo de estas vacunas, siendo aptas para la aplicación humana las que se encuentran en fase III. A la fecha del 10 de agosto de 2021 existen según (WHO, 2021) 184 candidatas a vacuna en fase preclínica y 110 candidatas a vacuna en diferentes fases clínicas. En la Fase III, se encuentran solamente 19 candidatas a vacunas, de las cuales, se ha seleccionado una muestra de 7 opciones para la evaluación. La selección de la muestra se hizo por conveniencia, los datos generales (nombre, tipo $\mathrm{y}$ dosis) de estas vacunas son retomados de (GOAL, 2021) y (WHO, 2021), los cuales se resumen en la Tabla 1. 
Tabla 1

Datos de las Vacunas Seleccionadas para el Estudio

\begin{tabular}{|c|c|c|c|c|c|}
\hline $\begin{array}{c}\text { Nombre } \\
\text { Fabricante }\end{array}$ & Tipo & Dosis & Efectos Secundarios & Efectividad & $\begin{array}{c}\text { Rango de } \\
\text { Precios }\end{array}$ \\
\hline $\begin{array}{l}\text { Pfizer, Inc., } \\
\text { BioNTech }\end{array}$ & BNT162b2 & 2 dosis & $\begin{array}{l}\text { Dolor, hinchazón, } \\
\text { enrojecimiento, } \\
\text { escalofríos, } \\
\text { dolor de cabeza, } \\
\text { cansancio. }\end{array}$ & $95 \%$ & $\begin{array}{c}\$ 7.00 \\
- \\
\$ 19.50\end{array}$ \\
\hline Moderna $T X$, Inc. & mRNA-1273 & 2 dosis & $\begin{array}{l}\text { Dolor, hinchazón, } \\
\text { enrojecimiento, } \\
\text { escalofríos, } \\
\text { dolor de cabeza, } \\
\text { cansancio. }\end{array}$ & $94 \%$ & $\begin{array}{c}\$ 15.00 \\
- \\
\$ 37.00\end{array}$ \\
\hline $\begin{array}{l}\text { AstraZeneca, } \\
\text { Universidad de } \\
\text { Oxford }\end{array}$ & ChAdOx1-S & 2 dosis & $\begin{array}{l}\text { Dolor, } \\
\text { fatiga, malestar general, } \\
\text { náuseas, mareos, dolor } \\
\text { de cabeza. }\end{array}$ & $63 \%$ & $\begin{array}{c}\$ 2.80 \\
- \\
\$ 13.30\end{array}$ \\
\hline $\begin{array}{l}\text { Sputnik } \boldsymbol{V}, \\
\text { Gamaleya Research } \\
\text { Institute, } \\
\text { Health Ministry } \\
\text { of the Russian } \\
\text { Federation }\end{array}$ & $\begin{array}{l}\text { Gam-COVID- } \\
\text { Vac Adeno-based } \\
\text { (rAd26-S+rAd5-S) }\end{array}$ & 2 dosis & $\begin{array}{l}\text { Dolor, } \\
\text { fatiga, malestar general, } \\
\text { náuseas, mareos, dolor } \\
\text { de cabeza. }\end{array}$ & $92 \%$ & $\begin{array}{c}\$ 3.00 \\
- \\
\$ 10.00\end{array}$ \\
\hline $\begin{array}{l}\text { Johnson \& Johnson } \\
\text { Janssen } \\
\text { Pharmaceutical }\end{array}$ & Ad26.COV2.S & 1 dosis & $\begin{array}{l}\text { Dolor, hinchazón, } \\
\text { enrojecimiento, } \\
\text { dolor de cabeza, } \\
\text { cansancio }\end{array}$ & $66 \%$ & $\begin{array}{c}\$ 8.50 \\
- \\
\$ 10.00\end{array}$ \\
\hline $\begin{array}{l}\text { Sinopharm, China } \\
\text { National Biotec } \\
\text { Group Co }+ \\
\text { Wuhan Institute of } \\
\text { Biological Products }\end{array}$ & $\begin{array}{l}\text { Inactivated SARS- } \\
\text { CoV-2 vaccine } \\
\text { (Vero cell) }\end{array}$ & 2 dosis & $\begin{array}{l}\text { Dolor, } \\
\text { fatiga, malestar general, } \\
\text { náuseas, mareos, dolor } \\
\text { de cabeza. }\end{array}$ & $19 \%$ & $\begin{array}{c}\$ 18.60 \\
- \\
\$ 44.00\end{array}$ \\
\hline $\begin{array}{l}\text { Sinovac, Research } \\
\text { and Development } \\
\text { Co., Ltd }\end{array}$ & $\begin{array}{l}\text { CoronaVac; } \\
\text { inactivated SARS- } \\
\text { CoV-2 vaccine } \\
\text { (vero cell) }\end{array}$ & 2 dosis & $\begin{array}{l}\text { Dolor, hinchazón, } \\
\text { enrojecimiento, } \\
\text { dolor de cabeza, } \\
\text { cansancio }\end{array}$ & $51 \%$ & $\begin{array}{c}\$ 10.30 \\
- \\
\$ 29.70\end{array}$ \\
\hline
\end{tabular}

Nota: Fuente propia de la investigación.

De forma más específica, los datos de efectos secundarios y efectividad mostrados en la tabla 1 , se han retomado de (CDC, 2021). Se aclara que se muestra la efectividad contra la enfermedad sintomática en la cepa original. No se muestra efectividad contra casos graves que requieren hospitalización, ni la efectividad contra variantes o cepas nuevas. Los datos de los precios se toman de (UNICEF, 2021) y muestran el rango de precios en dólares de Estados Unidos de Norteamérica de las vacunas en diferentes países.

De los datos de la Tabla 1, es claro que se pueden elegir dos estrategias:

1. Minimizar Costos: es evidente que para implementar esta estrategia bastará adquirir la mayor cantidad posible del tratamiento de AstraZeneca. 
2. Maximizar la Efectividad: en este caso, asumiendo que se desprecien los costos del tratamiento en favor de obtener los mejores resultados, se debería adquirir el tratamiento de Pfizer.

Para efectos del estudio, se requiere minimizar costos, por tal razón se selecciona de forma evidente el tratamiento de AstraZeneca (tomando en cuenta que su costo es relativamente bajo y su efectividad se mantiene viable). Para contestar la segunda pregunta, se ha desarrollado el modelo descrito a continuación.

El modelo epidemiológico SIR clásico posee tres estados: Susceptibles, Infectados y Recuperados, representados por $S$, $I$ y $R$ respectivamente. En $S$ tenemos individuos que aún no se han enfermado, pero si tienen contacto con un infectado pueden enfermarse. En $I$ se encuentran individuos en un estado infectado, es decir, enfermos e infecciosos, si contactan con individuos susceptibles pueden contagiarles la enfermedad. El estado $R$ de sujetos recuperados es un conjunto de individuos que se recuperaron o murieron y ya no son contagiosos.

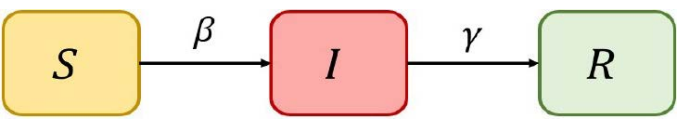

Figura 1. Modelo SIR Clásico.

Nota: Fuente propia de la investigación
Este modelo se puede representar gráficamente como se observa en la Figura 1.

Los datos más importantes al analizar una epidemia son $\beta$ que representa la tasa de transmisión del virus y $\gamma$ que representa la tasa de recuperación de los infectados. Los valores de $\beta$ y $\gamma$ en un modelo tradicional son fijos y la estimación de estos parámetros puede responder a diferentes metodologías. En este estudio, se usó un modelo más desarrollado que incluyó otras variables de estado que se consideran importantes al analizar el comportamiento del Covid-19, estas variables de estado son $E, I_{\text {pre }}, I_{\text {asin }}, I_{\text {sin }}, H$ y $F$ las cuales representan a los sujetos expuestos, infectados presintomáticos, infectados asintomáticos, infectados sintomáticos, hospitalizados y fallecidos respectivamente.

El modelo ha sido tomado de (McGee, 2021) el cuál se representa gráficamente en la Figura 2.

Las variables de estado que se incluyen en el modelo son:

$S: \quad$ Susceptibles.

$I_{\mathrm{pre}}$ : Infectados presintomáticos.

$I_{\text {asin }}:$ Infectados asintomáticos.

$I_{\text {sin }}:$ Infectados sintomáticos.

$H$ : Hospitalizados.

$R$ : Recuperados.

$F: \quad$ Fatalidades.

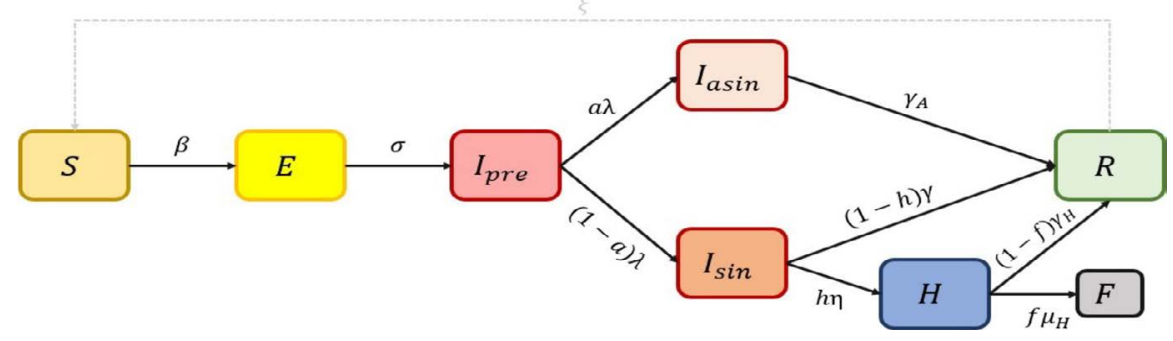

Figura 2: Modelo SEIR Extendido para el Covid-19.

Nota: Fuente propia de la investigación (2021). 
Las tasas de transición entre los estados se rigen por los parámetros:

$\lambda: \quad$ tasa de progresión a la infecciosidad (inverso del período latente).

$\gamma: \quad$ tasa de progresión a estado sintomático (inverso al período presintomático).

$a$ : probabilidad de que un individuo infectado permanezca asintomático.

$h$ : probabilidad de que un individuo sintomático sea hospitalizado.

$\eta$ : tasa de progresión al estado de hospitalización (inverso del período de inicio a ingreso).

$\gamma$ : tasa de recuperación para individuos sintomáticos no hospitalizados (inverso del período infeccioso sintomático).

$\gamma \boldsymbol{A}$ : tasa de recuperación para individuos asintomáticos (inverso del período infeccioso asintomático).

$\gamma H$ : tasa de recuperación de individuos sintomáticos hospitalizados (inverso del período infeccioso hospitalizado).

$f$ : $\quad$ probabilidad de muerte de las personas hospitalizadas (tasa de letalidad).

$\mu \mathrm{H}$ : tasa de muerte para los individuos hospitalizados (inverso del período de admisión hasta la muerte).

$\xi: \quad$ tasa de re-susceptibilidad (inverso del período de inmunidad temporal; 0 si es inmunidad permanente).

El efecto de las intervenciones o medidas de control (control por vacunación) se modeló mediante la introducción de compartimentos (estados del modelo) que representan a los individuos vacunados. Una persona puede ser vacunada cuando se encuentra en estado susceptible o expuesta al virus, aun así, puede mostrar síntomas de infección (ya que ninguno de los tratamientos es $100 \%$ efectivo), por lo cual, cada estado de enfermedad tiene un estado de vacunación correspondiente (con la excepción del estado de hospitalización, que se considera con un tratamiento diferente a la vacunación). Los individuos vacunados siguen la misma progresión a través de los estados de enfermedad, pero las tasas de transición u otros parámetros pueden ser diferentes (evidentemente estas tasas deberían ser más pequeñas). Puede ver el diagrama del modelo que incluye la vacunación en la Figura 3.

Además de los parámetros indicados anteriormente, las transiciones entre estados de vacunación se rigen por los parámetros:

$\beta_{V}:$ Tasa de transmisión para individuos vacunados.

$\sigma_{V}:$ tasa de progresión a la infecciosidad para individuos vacunados (inverso al período latente).

$\lambda_{V g}:$ tasa de progresión a (a) estado sintomático para individuos en cuarentena (inverso del período presintomático).

$\eta_{V}: \quad$ tasa de progresión al estado de hospitalización para los individuos en cua-

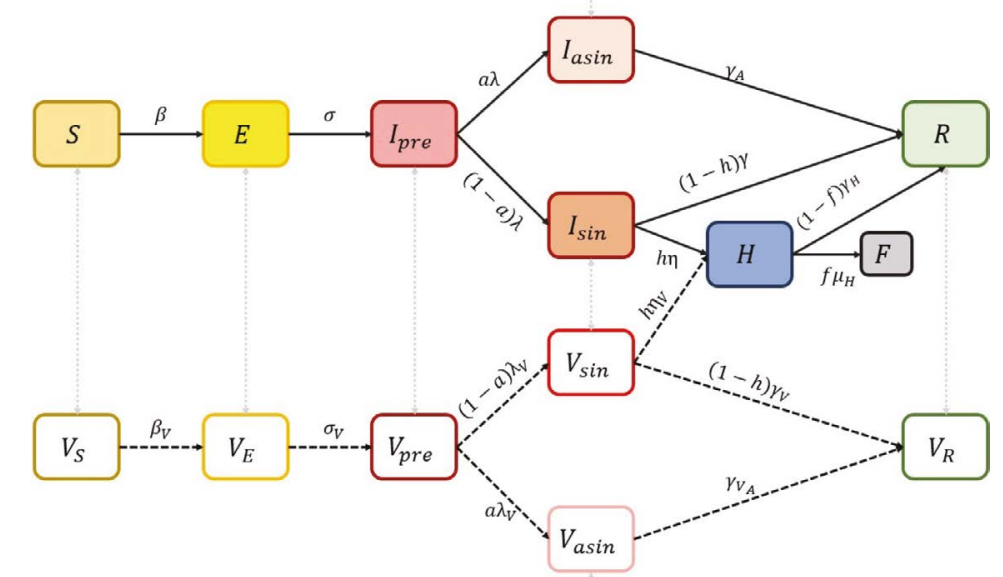

Figura 3. Modelo Extendido para el Covid-19 con Control por Vacunación.

Nota: Fuente propia de la investigación (2021). 
rentena (inverso del período de inicio a ingreso).

$\gamma_{V}$ : tasa de recuperación para individuos sintomáticos no hospitalizados que han sido vacunados (inverso del período infeccioso sintomático).

$\gamma_{V_{A}}$ : tasa de recuperación para individuos asintomáticos no hospitalizados (inverso del período infeccioso asintomático)

Para solucionar el modelo de la Figura 3, se pueden implementar diversos mecanismos, pero, en este estudio se utiliza un modelo de redes neuronales estocásticas. La idea general es modelar a través de una red de contactos las posibles transmisiones del virus para los escenarios de los individuos vacunados y los no vacunados.

Este modelo se implementa utilizando los datos demográficos de El Salvador siguiendo la siguiente línea:

1. Se define parámetros base como la cantidad de la población y la cantidad de personas expuestas iniciales a la enfermedad.

2. Se definen los parámetros de datos de la población salvadoreña, como distribución de edades, tamaño de los hogares, etc.

3. Se construyen las redes de contactos con las cuales se trabaja, una red de línea base (en la cual no hay intervenciones para la enfermedad) y una red que incluye el grupo etario vacunado.

4. Se establecen los parámetros del modelo SEIR, como las tasas de infección y de recuperación de la enfermedad.

5. Se configuran otros parámetros de interés, como datos de asintomáticos, protocolos de acción y cumplimiento de la intervención.
6. Se inicializa y se ejecuta el modelo para obtener resultados que se pueden analizar de forma gráfica.

Es necesario tomar en cuenta que alguna información de interés, como la frecuencia en la que se hacen pruebas de rastreo y pruebas aleatorias, porcentaje de contactos de casos primarios que se rastrean, número de días entre la aparición de los síntomas y el autoaislamiento de los infectados, número de días entre la administración de la prueba y el aislamiento de los casos positivos, número de días entre un contacto que se rastrea y el aislamiento de ese contacto y demás protocolos de acción, se toman en cuenta dentro del modelo. Además, los datos sobre el comportamiento de la pandemia se toman de los datos oficiales presentados por el Gobierno de El Salvador. Se puede revisar un ejemplo de la implementación del modelo en el siguiente enlace: implementación del modelo.

\section{Fase de Simulación}

Para las pruebas iniciales se define el tamaño de la población en $N=10,000$. También se define que los infectados iniciales por el virus rondan el $1 \%$ de la población. Para obtener la red de contactos, se ha utilizado un generador de red de comunidad demográfica definido en el paquete de Python seirplus_es. Esta función genera redes de contacto a nivel comunitario con propiedades de red realistas, según las estadísticas demográficas de la población de El Salvador. Los datos poblacionales se han obtenido de la Encuesta de Hogares de Propósitos Múltiples de El Salvador (DIGESTYC, 2018).

Cada nodo de la red simula un sujeto de la población. En la Figura 4, se muestra la distribución de los nodos en una red 
generada con los datos de contacto de El Salvador. Esta red de contactos sería la distribución general sin intervención de tratamiento. Tome en cuenta que esta red en particular tiene los siguientes parámetros:

- $\quad$ Estadísticos: media $=14.43, \mathrm{std}=12.76$, $\mathrm{CV}=0.88, \mathrm{CI}=(3,749) 95 \%$

- $\quad$ Asortatividad: 0.17

- Clustering coeff: 0.28

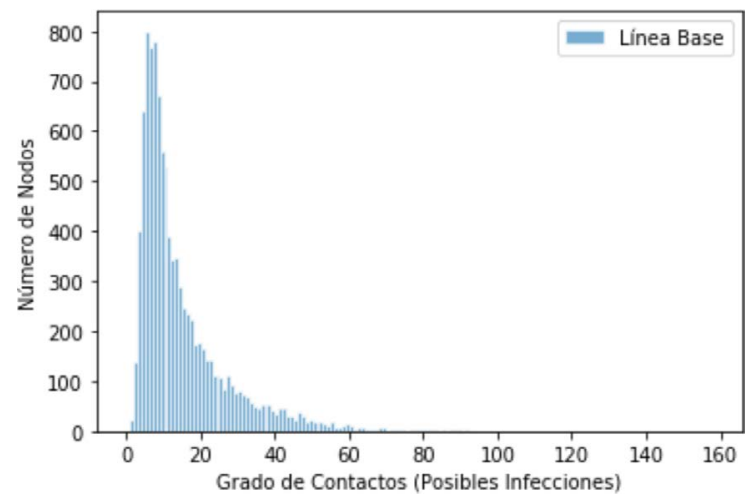

Figura 4. Distribución de Nodos de Red de Contactos.

Nota: Fuente propia de la investigación

Los valores de los parámetros se asignan a los miembros de la población de forma individual. Se generan distribuciones de valores para cada parámetro, y algunos de estos valores se especifican de manera estratificada por edad, modelando así una población realista heterogénea. Por ejemplo, las distribuciones para el período de latencia, período presintomático y el período de incubación total de la enfermedad se muestran en la gráfica de la Figura 5.

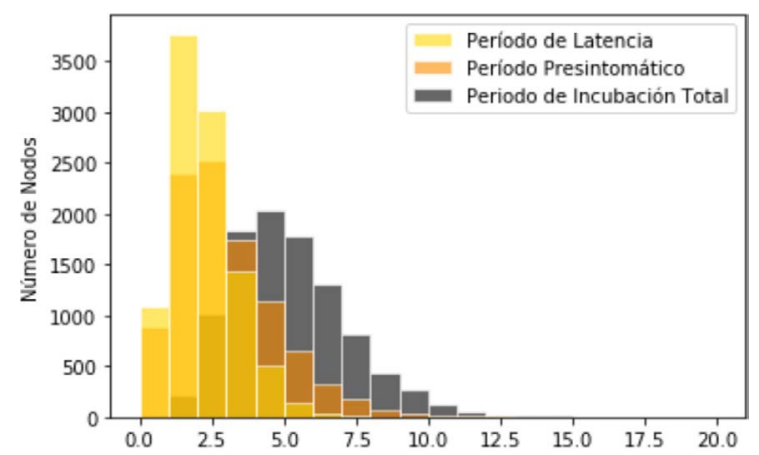

Figura 5. Distribución de Períodos de la Infección.

Nota: Fuente propia de la investigación

El número reproductivo básico promedio $R_{0}$ del Covid-19 se ha tomado como 2.76 con un coeficiente de variación de 0.2 (Argueta, 2020). La distribución para $R_{0}$ se muestra en la gráfica de la Figura 6.

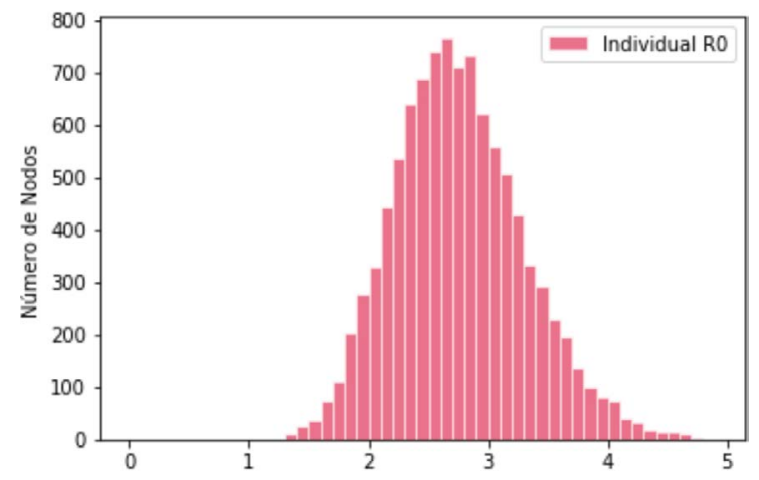

Figura 6: Distribución de $R_{0}$ en El

Salvador.

Nota: Fuente propia de la investigación

De aquí que $\beta=R_{0} /$ Período de Infección y $\beta v=\beta\left(0.1 / R_{0}\right)$. Los valores de $\alpha$ se diferencian por grupos de edades: $\alpha=0.5$ para edades entre 0 y 19 años y $\alpha=1$ para edades mayores a los 19 años. Además, se define $p=0.2$ para reflejar que el $20 \%$ de las interacciones son con contactos incidentales o casuales fuera de su conjunto de contactos cercanos y $q=0.05$ para reflejar las interacciones de los vacunados con posibles 
contagios. También, se toma el porcentaje de asintomáticos como un $33 \%$, basado en datos de (GOES, 2021).

Una vez definidos los parámetros de interés, se puede correr el modelo, el cuál presenta resultados gráficos. Por ejemplo, si se toman como vacunados solamente los grupos de riesgo (mayores de 60 años) y se mantiene una política de distanciamiento social para el $30 \%$ de la población Línea Base, se obtienen resultados como el mostrado en la Figura 7, donde se indican porcentajes de población infectada.

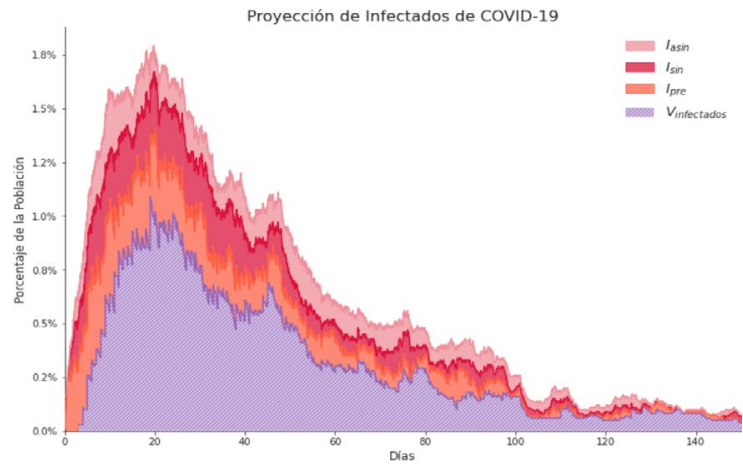

Figura 7. Resultados de la Simulación del Modelo.

Nota: Fuente propia de la investigación

\section{Fase de Optimización}

Para desarrollar la fase de optimización, se aplicarán diversas políticas y se verán los resultados de estas políticas en el modelo. Luego se analizarán estos resultados y se seleccionará la estrategia que minimice el impacto de la pandemia. De forma inmediata se definen dos tipos de políticas:

1. Distanciamiento social.

2. Vacunación.

El distanciamiento social se implementa en el modelo a través de la red de contactos, esto delimita los contactos de cada nodo. Por otra parte, existirán diversos tipos de vacunación, específicamente se analizarán dos posibles casos: Vacunar a los grupos de riesgo (mayores de 60 años) y vacunar a la población económicamente activa (con edades entre 20 y 50 años). Con estas especificaciones, resultan los siguientes posibles escenarios:

1. No se aplica distanciamiento social y se vacuna la población vulnerable.

2. No se aplica el distanciamiento social y se vacuna la población económicamente activa.

3. Se aplica el distanciamiento social y se vacuna la población vulnerable.

4. Se aplica el distanciamiento social y se vacuna la población económicamente activa.

Para la evaluación de estos escenarios se toman en cuenta dos criterios: la efectividad y el costo de implementar las políticas establecidas. La efectividad se calcula mediante el siguiente indicador:

$$
\text { Efect }_{i}=\left(\frac{\operatorname{In} f_{i}}{\operatorname{In} f_{0}}\right) * 100
$$

Donde:

- $\quad$ Efect: Efectividad de la estrategia $i$, con $i \hat{\mathrm{I}}[1,4]$

- $\quad \operatorname{Inf} f_{0}$ Infectados que resultan sin aplicar ninguna estrategia.

- $\quad$ Inf: Infectados que resultan al aplicar la estrategia $i$.

Tome en cuenta que se debe cumplir la condición: $\operatorname{Inf} f_{\mathrm{i}} \leq \operatorname{Inf} f_{0}$. Además, gráficamente se representa $\operatorname{Inf} f_{0}$ por el color rojo y, por otra parte, $I n f_{\mathrm{i}}$ se representa por color violeta, este dato será importante para el 
análisis visual de las gráficas presentadas. El costo de cada estrategia $i$ se evaluará con la siguiente fórmula:

$$
C_{T}(i)=C_{d s}(i)+C_{V}(i)
$$

Donde:

- $\quad C_{\mathrm{T}}(i)$ : Costo total de la $i$-ésima estrategia.

- $\quad C_{\mathrm{ds}}(i)$ : Costo de distanciamiento social de la $i$-ésima estrategia.

- $\quad C_{\mathrm{v}}(i)$ : Costo de vacunación de la $i$-ésima estrategia.

El costo del distanciamiento social se toma como referencia del análisis propuesto en (Thunström et al. 2020). De forma concreta, el costo del distanciamiento social se tomará constante como el $6.2 \%$ del PIB del país. El PIB nominal de El Salvador en 2019 fue de \$ 28.989 millones (FMI, 2020), el costo estimado del distanciamiento social será:

$$
C_{\mathrm{ds}}(i)=28.989 * 0.062 \approx \$ 1.8 \text { millones }
$$

Si no hay distanciamiento social entonces $C_{\mathrm{ds}}(i)=0$. En concreto, por cada estrategia se calcula su efectividad y su costo y con estos dos criterios se selecciona la estrategia óptima. Tome en cuenta que este valor es estimado y se usará solamente como referencia. No se valoran costos de transporte, distribución o almacenamiento. Además, este indicador se usa solamente para fines de comparación en este estudio, no se debe confundir con los costos reales para la administración del tratamiento.

\section{Resultados}

\section{Estrategia 1: No aplicar distancia- miento social, pero vacunar la po- blación vulnerable}

Se implementan en el modelo dos ajustes, se le indica que no debe aplicar distanciamiento social para la población y que se van a vacunar solamente las personas mayores a 60 años. Con estos ajustes, los resultados se muestran en la Figura 8.

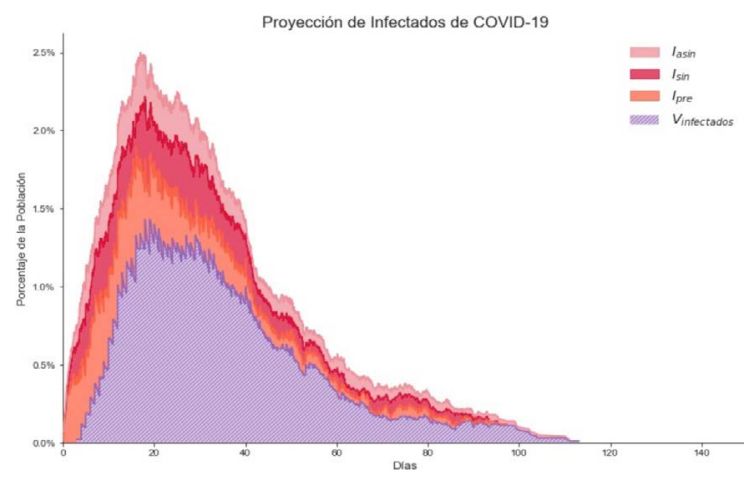

Figura 8. Proyecciones de Infectados Aplicando la Estrategia 1.

Nota: Fuente propia de la investigación.

Para el caso específico de la estrategia 1, se tiene que su efectividad es:

$$
\text { Efect }_{1}=\left(\frac{1.4}{2.3}\right) * 100=60.86 \%
$$

Para calcular el respectivo costo, se procede como sigue: La proporción de población vulnerable es del $14.1 \%$ según datos poblacionales obtenidos de (DIGESTYC, 2018), por lo cual se necesitarían administrar un total aproximado de 0.9 millones de vacunas. Y el costo de la vacuna AstraZeneca (tratamiento que minimiza los costos) tiene un valor estimado de $\$ 2.8$ (precios sujetos a cambios). Con esta información, el costo de vacunación es:

$$
C_{\mathrm{T}}(1)=0+0.9 * \$ 2.8 \approx \$ 2.52 \text { millones }
$$




\section{Estrategia 2: No aplicar distancia- miento social, pero vacunar la po- blación económicamente activa.}

En esta estrategia se le indica al modelo que no se guarda distanciamiento social, pero se vacuna la población que corresponde a los grupos de edad de entre $20 \mathrm{y}$ 59 años. Los resultados de estos ajustes se muestran en la Figura 9.

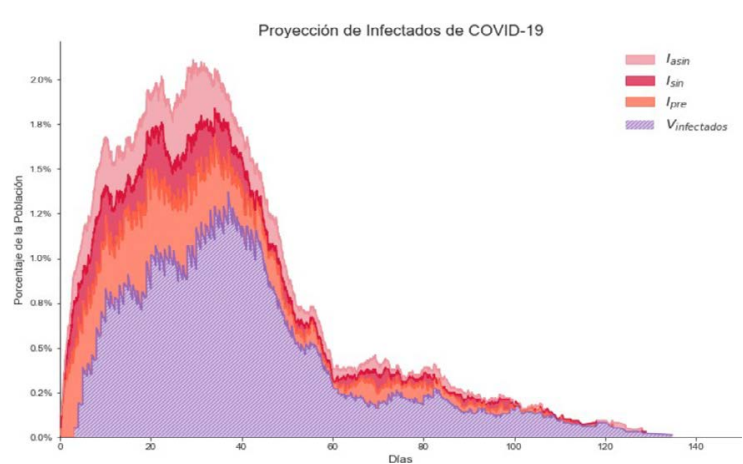

Figura 9. Proyecciones de Infectados Aplicando la Estrategia 2.

Nota: Fuente propia de la investigación.

Su efectividad es:

$$
\text { Efect }_{2}=\left(\frac{1.4}{1.9}\right) * 100=73.68 \%
$$

Por otra parte, para calcular el costo, se estima que el $56.1 \%$ de la población recibiría vacuna, este porcentaje equivale aproximadamente a 3.5 millones de habitantes que serían vacunados, así el resultado será:

$$
C_{\mathrm{T}}(2)=0+3.5 * \$ 2.8 \approx \$ 9.8 \text { millones }
$$

Estrategia 3: Se aplica el distanciamiento social y se vacuna la población vulnerable

Se evalúa el caso en el cual además de vacunar grupos vulnerables, se aplican medidas de distanciamiento social (restricción de la movilidad o cuarentena, por ejemplo).
Los resultados de esta estrategia se muestran en la Figura 10.

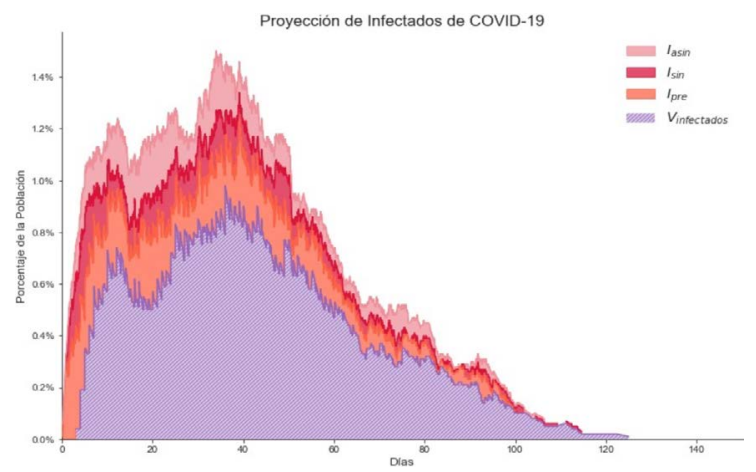

Figura 10. Proyecciones de Infectados Aplicando la Estrategia 3.

Nota: Fuente propia de la investigación.

Su efectividad es

$$
\text { Efect }_{3}=\left(\frac{1.3}{1.9}\right) * 100=68.42 \%
$$

Y el costo de aplicar esta estrategia se deduce como:

$$
C_{\mathrm{T}}(3)=\$ 1.8+0.9 * \$ 2.8 \approx \$ 4.32 \text { millones }
$$

Estrategia 4: Se aplica el distanciamiento social y se vacuna la población económicamente activa

En este caso se aplican políticas de distanciamiento social y además vacunación a la población económicamente activa. Los resultados se ven en la Figura 11.

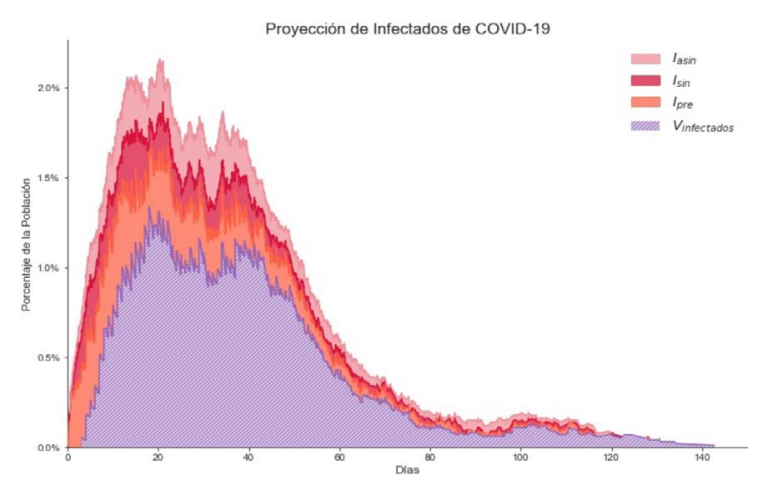

Figura 11. Proyecciones de Infectados Aplicando la Estrategia 4.

Nota: Fuente propia de la investigación. 
La efectividad de esta estrategia es:

$$
\text { Efect }_{4}=\left(\frac{1.0}{1.3}\right) * 100=76.92 \%
$$

Y el costo de aplicar esta estrategia se deduce como:

$$
C_{\mathrm{T}}(4)=\$ 1.8+3.5 * \$ 2.8 \approx \$ 11.6 \text { millones }
$$

\section{Evaluación de las Estrategias}

Se presentan los resultados de la evaluación de las diferentes estrategias en la tabla siguiente:

Tabla 2.

Resumen de Criterios Evaluación de Estrategias

\begin{tabular}{ccc}
\hline Estrategia & Efectividad & Costo \\
\hline $\mathbf{1}$ & 60.86 & 2.52 \\
$\mathbf{2}$ & 73.68 & 9.8 \\
$\mathbf{3}$ & 68.42 & 4.32 \\
$\mathbf{4}$ & 76.92 & 11.6 \\
\hline
\end{tabular}

Nota: Fuente propia de la investigación.

Para seleccionar la mejor estrategia se desarrollarán los siguientes pasos:

1. Se estandarizan los datos de la tabla 5. Para ello, los valores de la tabla se transforman mediante la siguiente fórmula:

$$
Z_{i j}=\frac{x_{i j}-\min \left(x_{j}\right)}{\max \left(x_{j}\right)-\min \left(x_{j}\right)}
$$

donde $i$, representa la fila de cada dato y $j$ su respectiva columna.

2. Se mantendrán los valores positivos para la efectividad, pero el costo se convierte en valores negativos.

3. El resultado de la evaluación para cada estrategia será medido con la fórmula:

$$
E_{\mathrm{i}}=\text { Efect }_{\mathrm{i}}+C(i)
$$

4. Tomar decisión: elegir la estrategia con mejor puntaje (bajo la relación efectividad-costo).

Al aplicar estas transformaciones a la Tabla 2, se obtiene el siguiente resultado:

Tabla 3.

\section{Evaluación de las Estrategias}

\begin{tabular}{cccc}
\hline Estrategia & $\begin{array}{c}\text { Efectividad } \\
\text { Estandarizada }\end{array}$ & $\begin{array}{c}\text { Costo } \\
\text { Estandarizado }\end{array}$ & $\begin{array}{c}\text { Resultado de } \\
\text { la Evaluación }\end{array}$ \\
\hline $\mathbf{1}$ & 0.00 & 0.00 & 0.00 \\
$\mathbf{2}$ & 0.80 & -0.80 & 0.00 \\
$\mathbf{3}$ & 0.47 & -0.20 & 0.27 \\
$\mathbf{4}$ & 1.00 & -1.00 & 0.00 \\
\hline
\end{tabular}

Nota: Fuente propia de investigación.

Al observar los resultados obtenidos, la estrategia mejor evaluada es la estrategia 3 , por lo tanto, se considera que la mejor opción bajo el criterio efectividad-costo es aplicar el distanciamiento social y vacunar la población vulnerable.

Con los elementos desarrollados en esta sección, ya se cuenta con insumos suficientes para desarrollar algunas conclusiones y recomendaciones de interés.

\section{Conclusiones}

Es necesario tomar en cuenta que el modelo analiza gran cantidad de variables y elementos, algunos de ellos tienen naturaleza estocástica (como las redes de contactos), por lo cual, reproducir de forma constante valores exactos es muy poco probable. Es decir, el modelo no es determinista. Sin embargo, bajo las condiciones que se le impongan, los resultados que se obtengan convergerán a valores similares.

Si bien es cierto, el desarrollo del proyecto se centra en un sistema bien definido y delimitado, el proceso que se desarrolla en 
la investigación se puede replicar y aplicar para el análisis de cualquier otro sistema de la misma naturaleza. Justamente esa replicabilidad hace interesante el análisis de modelos de este tipo, ya que se puede aplicar el mismo proceso aquí descrito a otros niveles, por ejemplo, se puede analizar el comportamiento del virus a nivel local en un entorno pequeño, o se puede aplicar a nivel general con datos poblacionales de cualquier país.

Después de analizar múltiples variables y diversas estrategias, se llega a una conclusión lógica respaldada por el análisis exhaustivo de este estudio: la estrategia óptima para distribuir la vacuna covid19 es aplicar el tratamiento a personas vulnerables y, además, mantener medidas de distanciamiento social. Es la mezcla de estas dos políticas que genera los mejores resultados para disminuir o erradicar la pandemia. También, tomar en cuenta que la cantidad mínima de vacunas que se deben obtener para desarrollar esta estrategia es de aproximadamente 0.9 millones.

El modelo muestra un resultado contundente: a pesar de que se aplique un tratamiento (vacuna) en una población, no se pueden omitir las medidas de distanciamiento social. De esta manera, nace la necesidad de concientizar a la población de no abandonar las medidas necesarias, ya que la vacuna representa no una solución definitiva para erradicar el virus.

Estas conclusiones son válidas con variables, parámetros y datos poblacionales de El Salvador, ya que con estos datos se ha ajustado el modelo desarrollado.

\section{Financiamiento}

Universidad Gerardo Barrios, El Salvador. INV021-2021.

\section{Conflicto de intereses}

El autor declara no tener algún conflicto de interés.

\section{Declaración de la contribución de los autores}

El porcentaje total de contribución para la conceptualización, preparación y corrección de este artículo fue el siguiente: V.L.S. $100 \%$.

\section{Declaración de disponibilidad de los datos}

Los datos que respaldan los resultados de este estudio serán puestos a disposición por el autor correspondiente V.L.S., previa solicitud razonable.

\section{Referencias}

Acuña Soto, R., Castañeda Dávila, L. \& Chowe1l, G. (2011). A perspective on the 2009 A/ H1N1 influenza pandemic in Mexico. Mathematical Biosciences and Engineering, MBE, 8(1), 223-238. https://doi.org/10.3934/ mbe.2011.8.223

Argueta, C. E. (2020). El COVID-19 y el número reproductivo básico y efectivo en El Salvador: Una propuesta para su medición. FUNDAUNGO. https://www.fundaungo. org.sv/products/el-covid-19-y-el-numero-reproductivo-basico-y-efectivo-en-el-salvador-una-propuesta-para-su-medicion/492

Arino, J., Brauer, F. \& den Driessche, P. (2018). A model for influenza with vaccination and antiviral treatment. Journal of theoretical biology, 253(1), 118-130. https://doi.org/10.1016/j.jtbi.2008.02.026

Brauer, F., Castillo-Chávez, C., Pava Salgado, E., \& Barley, K. (2015). Modelos de la propagación de enfermedades infecciosas. Universidad Autónoma de Occidente. https://doi. org/10.13140/2.1.4882.5929 
CDC. (2021). Diferentes vacunas contra el COVID-19. Centers for Disease Control and prevention. https:/espanol.cdc.gov/coronavirus/2019-ncov/ vaccines/different-vaccines.html

Chowell, G., Ammon, C., Hengartner, N. \& Hyman, J. (2006). Transmission dynamics of the great influenza pandemic of 1918 in Geneva, Switzerland: Assessing the effects of hypothetical interventions. Journal of theoretical biology 241(2), 193-204. https://doi.org/10.1016/j. jtbi.2005.11.026

Dal Molin Ribeiro, M.H., Gomes Da Silva, R., Cocco Mariani, V. \& Dos Santos Coelho, L. (2020). Short-term forecasting COVID-19 cumulative confirmed cases: Perspectives for Brazil. Chaos, Solitons and Fractals. https:// doi.org/10.1016/j.chaos.2020.109853

DIGESTYC. (2018). Encuesta de Hogares de Propósitos Múltiples 2018. Dirección general de estadística y censos. http:/www. digestyc.gob.sv/index.php/novedades/avisos/869-ya-se-encuentra-disponible-la-publicacion-ehpm-2018.html

FMI. (2020). World economic outlook databases. International Monetary Fund. https:/www.imf. org/en/Publications/SPROLLs/world-economic-outlook-databases\#sort $=\% 5 \mathrm{C} \% 40 \mathrm{imfda}$ te $\% 5 \mathrm{C}$ descending

GOAL. (2021). ¿Cuánto cuesta la vacuna contra el COVID-19? https:/www.goal.com/es-mx/ noticias/cuanto-cuesta-vacuna-covid-19/1d0ngem2skf521hey98yr7shru

GOES. (10 de agosto, 2021). Datos diarios de COVID 19 en El Salvador. https://covid19.gob.sv/

Gómez Marín, J. (2020). Una hoja de ruta para la Vacuna COVID 19 en Colombia, un reto posible. Infectio. http://dx.doi.org/10.22354/ in.v25i1.901

González-Melado, F., \& Di Pietro, M. L. (2020). La vacuna frente a la COVID-19 y la confianza institucional. Enfermedades Infecciosas y Microbiología Clínica. https://doi. org/10.1016/j.eimc.2020.08.001

Herrera, M., Cruz, M. \& Castillo-Chavez, C. (2011). Multiple outbreaks for the same pandemic: Local transportation and social distancing explain the different 'waves' of A-H1N1pdm cases observed in México during 2009. Mathematical biosciences and engineering: MBE, 8(1), 21-48. https://doi.org/10.3934/ mbe.2011.8.21
Lenhart, S., \& J. Workman. (2007). Optimal control applied to biological models.

McGee, S. (2021). SEIRS+ Model Framework. https://github.com/ryansmcgee/seirsplus/wiki

Nuño, M., G. Chowell, \& C. Castillo-Chavez. (2007). On the role of cross-immunity and vaccines on the survival of less fit flu-strains. Theorical Population Biology 71(1), 20-29. https://doi.org/10.1016/j.tpb.2006.07.002

OPS. (2020). Fases de desarrollo de una vacuna. Organización Panamericana de la Salud. https://www.paho.org/es/documentos/ covid-19-fases-desarrollo-vacuna

Piqueras, M., Cruz, Hortal Carmona, J. \& Padilla Bernáldez, J. (2020). Visteme despacio que tengo prisa. Un análisis ético de la vacuna del COVID-19: fabricación, distribución y reticencia. Enrahonar. An International Journal of Theoretical and Practical Reason 65, 57-73. https://ddd.uab.cat/pub/enrahonar/enrahonar a2020v65/enrahonar_a2020v65p57.pdf

Chimmula, R., Kumar, V. \& Zhang, L. (2020). Time series forecasting of COVID-19 transmission in Canada using LSTM networks. Chaos, Solitons and Fractals, 135. https://doi.org/10.1016/j.chaos.2020.109864

Saralegui Vallejo, U. (2016). Modelos epidémicos con control por vacunación. https:/www.researchgate.net/publication/322273233_Modelos_epidemicos_con_control_por_vacunacion

Thunström, L., Newbold, S., Finnoff, D., Ashworth, M., \& Shogren, J. (2020). Beneficios y costos de usar el distanciamiento social para aplanar la curva de COVID-19. Journal of Benefit-Cost Analysis. http://ebour. com.ar/pdfs/Beneficios $\% 20 y \% 20 \operatorname{costos} \% 20$ de $\% 20$ usar $\% 20$ el $\% 20$ distanciamiento $\% 20$ social $\% 20$ para $\% 20$ aplanar $\% 201$ a $\% 20$ curva $\% 20 \mathrm{de} \% 20$ COVID $19 . p d f$

UNICEF. (2021). COVID-19 Vaccine Market Dashboard. https://www.unicef.org/supply/covid-19-vaccine-market-dashboard .

WHO. (2021). Draft landscape and tracker of COVID-19 candidate vaccines. https://www. who.int/publications/m/item/draft-landscape-of-covid-19-candidate-vaccines. 


\section{(c) $(1)(9) \Theta$}

Distribución de vacuna COVID-19: Combinando SEIR y Machine Learning (Víctor López-Sandoval) Uniciencia is protected by Attribution-NonCommercial-NoDerivs 3.0 Unported (CC BY-NC-ND 3.0) 